\title{
Crioconservação de espécies de Amaryllidaceae ${ }^{(1)}$
}

\author{
ANTONIO FERNANDO CAETANO TOMBOLATO ${ }^{(2,3)}$, THIAGO NOGUEIRA LUCON(2,4), MARA FERNANDES MOURA ${ }^{(2)}$, \\ WILSON BARBOSA ${ }^{(2,3)}$, MAURO BRUM MONTEIRO JÚNIOR ${ }^{(2,3)}$, YARA SCHIAVINATO ${ }^{(2,4)}$ E RENATO FERRAZ DE ARRUDA VEIGA ${ }^{(2)}$
}

\section{RESUMO}

O Jardim Botânico do IAC tem como objetivo conservar as espécies nativas do Estado de São Paulo, oferecendo áreas para a preservação in situ, ex situ e in vitro, além de câmaras com baixa temperatura, já que a crioconservação se transformou também em alternativa interessante para a conservação de recursos genéticos. Realizaram-se testes de crioconservação em sementes recalcitrantes de três espécies de Amaryllidaceae: Hippeastrum papilio (HP), Hippeastrum glaucescens (HG) e Zephyranthes robusta (ZR). No delineamento experimental, foram estabelecidas quatro repetições de 25 sementes cada, em 4 tratamentos: T1: $-196^{\circ} \mathrm{C}$ sem protetor; $\mathrm{T} 2:-196^{\circ} \mathrm{C}$ com o protetor PVS2; T3: $-196^{\circ} \mathrm{C}$ com o agente protetor de alginato de sódio; T4: semeadura direta sem nenhum tratamento a frio (testemunha). As porcentagens de germinação obtidas foram: T1: 98\%, 70\% e 80\%, respectivamente para ZR, HP e HG. T2: - 93\%, 68\% e 21\%, respectivamente. T3: - 1\%, 0\% e 8\% respectivamente; T4: 99\%, $72 \%$ e $88 \%$, respectivamente. A partir dos resultados, pode-se observar que os agentes protetores não foram necessários para a conservação das sementes nas condições do ensaio, uma vez que as taxas de germinação foram melhores sem PVS2 ou alginato de sódio. Observou-se também que a crioconservação sem agentes protetores, propiciou a germinação mais rápida do que a por testemunha para as três espécies testadas. Verificou-se uma provável quebra de dormência da semente de ZR, pois, houve uma aceleração no processo de germinação de cerca de dois dias. Esta observação merece maior aprofundamento.

Palavras-chave: Nitrogênio líquido, conservação, sementes, recursos genéticos.

\section{ABSTRACTS}

\section{Crioconservation of Amaryllidaceae Species}

The IAC Botanical Garden has as objective to conserve native species of the State of São Paulo, and it has preservation areas for in situ, ex situ and in vitro, beyond chambers with low temperature once the crioconservation has become an interesting alternative for the conservation of genetic resources. The Amaryllidaceae family presents plants of high ornamental value and great commercial appeal and, for these reasons, it has been object of attention for the conservation of its natural diversity. An experiment with 3 species of recalcitrant seeds of Amaryllidaceae was carried through: Hippeastrum papilio (HP), Hippesatrum glaucescens (HG) and the Zephyrantes robusta (ZR), being 4 treatments with four repetitions of 25 seeds each. The treatments were: T1. -196OC without protector; T2. -196OC with the protector PVS2. T3. -196OC with the protector agent sodium alginate; T4. seeds sown directly with no cold treatment (control). The obtained data are: T1 - ZR, HP and HG showed 98\%, 70\% and $80 \%$ of germination, respectively. T2 $-93 \%, 68 \%$ and $21 \%$, respectively. T3 $-1 \%, 0 \%$ and $8 \%$, respectively. Finally, T4 - 99\%, $72 \%$ and $88 \%$, respectively. The results prove that the protective agents are not necessary for these species, once germination rates were better without PVS2 or sodium alginate. It was also observed that the crioconservation, without protector agent, showed the seed germination earlier than the control, then it is possible to imagine that a break of seed dormancy occurred. This concept must be confirmed.

Keywords: liquid nitrogen, conservation, seeds, genetic resources

\section{INTRODUÇÃO}

\subsection{Crioconservação}

A crioconservação é uma alternativa eficiente e prática para a conservação de germoplasma em longo prazo, especialmente em se tratando de espécies que apresentam sementes recalcitrantes ou intermediárias (SANTOS, 2000). Essa técnica se caracteriza pela conservação de uma estrutura vegetal a temperaturas ultrabaixas $\left(-196^{\circ} \mathrm{C}\right.$ a $\left.-150^{\circ} \mathrm{C}\right)$, obtidas pela utilização do nitrogênio líquido, paralisando o metabolismo e impedindo a deterioração fisiológica do material biológico (GONZÁLEZ-BENITO, 1998).
Atualmente, muitas coleções estão se perdendo devido à falta de recursos financeiros, pois seu custo de manutenção, tanto in situ quanto in vitro, é elevado. A crioconservação, quando comparada às outras formas de conservação, é mais barata e de mais fácil controle, além de não estar sujeita a pragas e doenças, como acontece na conservação em campo. Assim, se o nitrogênio líquido for mantido em nível adequado, a amostra permanece conservada (ENGELMANN, 1991).

Para a conservação de sementes, é necessário o conhecimento prévio de algumas características. A identificação correta de seu comportamento em relação à armazenagem

\footnotetext{
(1) Recebido para publicação em 25/1/2007 e aceito em 19/1/2009.

(2) Instituto Agronômico de Campinas, IAC, NPD - Jardim Botânico, Caixa Postal 28, 13012-970, Campinas (SP), Brasil. tombolat@iac.sp.gov.br

(3) Bolsista CNPq

(4) Bolsista Fapesp
} 
faz-se necessária para a escolha da estratégia adequada na conservação, sendo o teor de água das sementes um fator crítico nessa identificação. (FONSECA, 2003). Antes da crioconservação, as sementes ortodoxas eram as únicas que podiam ser conservadas durante longo prazo nos bancos de germoplasma. Sementes que têm comportamento ortodoxo são as que toleram a desidratação a teores de água entre $2 \%$ e $5 \%$ de umidade sem qualquer dano, e, além disso, podem ser armazenadas em baixas temperaturas, o que aumenta seu período de conservação, sem que percam a viabilidade. Já as sementes recalcitrantes não toleram a dessecação (no caso de redução de umidade, perdem a viabilidade), devendo, assim, ser armazenadas com alto grau de umidade, o que dificulta sua conservação. No entanto, mesmo em condições úmidas, essas sementes têm curta longevidade, que varia de poucas semanas a alguns meses. (ROBERTS, 1973).

Convém salientar que a crioconservação ainda precisa ser estudada para muitas espécies, incluindo as ornamentais, cujos protocolos disponíveis se concentram em banana e citros, segundo SANTOS (2000). Em muitas espécies de plantas ornamentais observam-se sementes intermediárias, tolerantes à desidratação em níveis relativamente baixos, mas que são danificadas por temperaturas abaixo de zero quando secas (ELLIS et al., 1990). Há, também, espécies recalcitrantes nas quais não é possível a desidratação a níveis de umidade suficientemente baixos, que possibilite seu armazenamento em temperaturas negativas. Nesse caso, mesmo com o avanço das técnicas de conservação para uso imediato em BAGs (Bancos Ativos de Germoplasma), verifica-se que essas espécies ainda são muito sensíveis ao congelamento (SANTOS, 2000). Outra dificuldade comum aos BAGs é a manutenção de genótipos distintos em um único local, já que, por serem submetidos a estresse fisiológico - especialmente os exóticos -, perdem seu vigor natural ou morrem suas plantas (SANTOS, 2000).

No método da crioconservação - à temperatura de -196 ${ }^{\circ} \mathrm{C}$ - todos os processos metabólicos, como respiração e atividades enzimáticas, são inativos. Há um pequeno risco, que pode ser contornado, de danos cumulativos, resultantes da atividade de radicais livres induzidos por radiação background. Em geral, no material armazenado por crioconservação, não há mudanças significativas em função do tempo (ASHWOOD-SMITH, 1985); nesse processo, os problemas associados à manutenção de culturas são eliminados. O único dispêndio necessário é com o reabastecimento de nitrogênio líquido.

Apesar das vantagens da crioconservação, existem problemas decorrentes da complexidade técnica e biológica do processo de congelamento e descongelamento, já que são verificadas reações diferentes de cada espécie, o que exige o desenvolvimento de procedimentos específicos para cada tipo de cultura. No entanto, esse método ainda é mais adequado do que o crescimento lento provocado por grande elenco de sistemas de cultura, como, por exemplo, a técnica de manutenção in vitro sob baixa temperatura e em meio de cultura mínimo (com baixos teores de nutrientes). Acredita-se que, com o avanço dos conhecimentos sobre esse tema, novos e melhores métodos, mais generalizados, surgirão (TORRES; CALDAS \& BUSO, 1998).
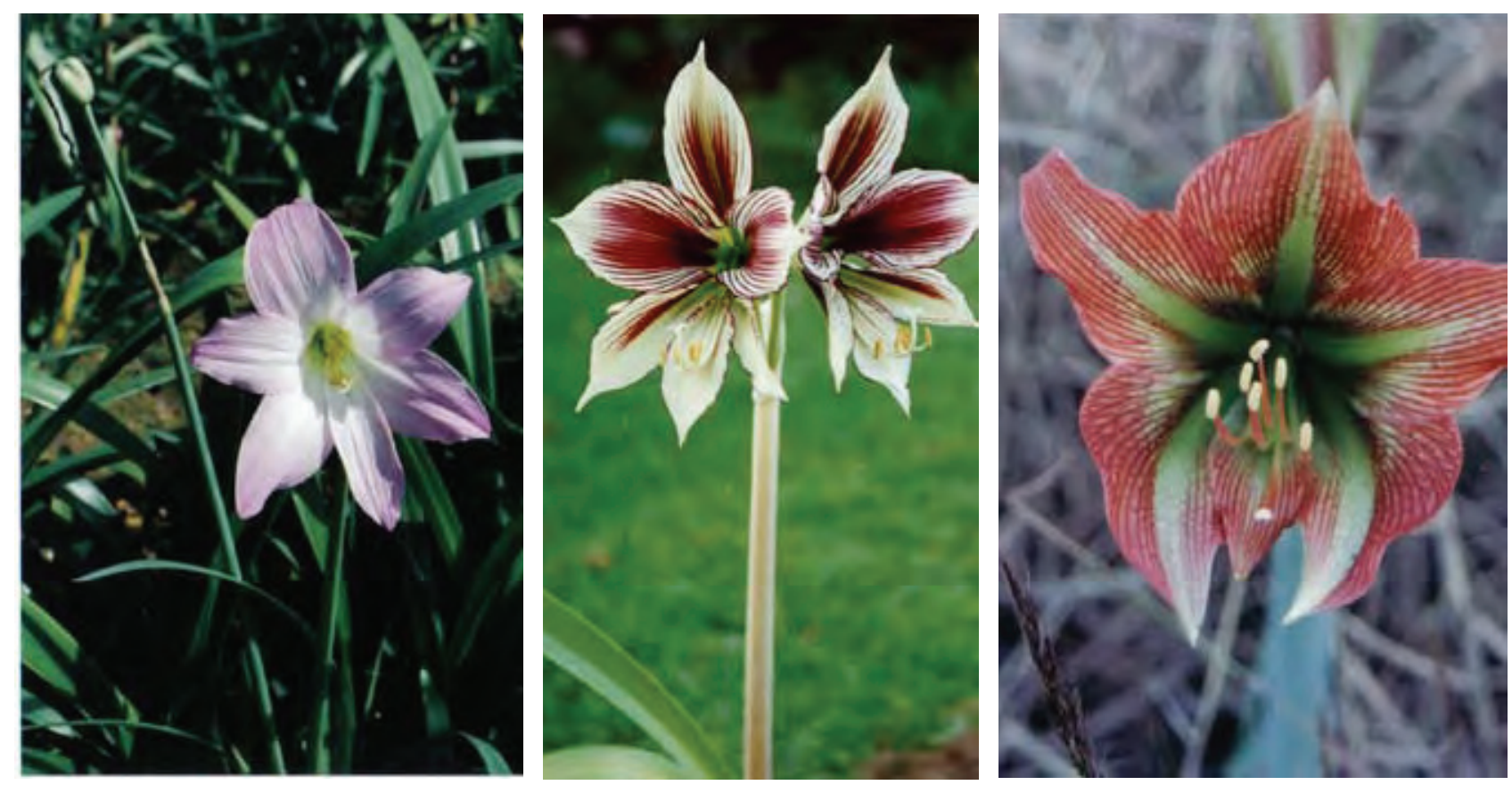

Figura 1. A. Zephyranthes robusta. B. Hippeastrum papilio. C. Hippeastrum glaucescens 


\subsection{As espécies de Amaryllidaceae}

O objeto de estudo deste experimento são três espécies bulbosas nativas brasileiras, da família Amaryllidaceae: Zephyranthes robusta, Hippeastrum papilio e Hippeastrum glaucescens.

Os lírios da chuva (Zephyranthes robusta) (figura 1A), nomeados assim por causa de sua tendência de abrir as flores após a chuva, são originários do Brasil, principalmente da região Sul; são plantas perenes, de pequeno porte, que florescem em diversas estações. Entre as bulbosas, são as de mais fácil cultivo, sendo, muitas vezes, usadas em paisagismo de jardins. Crescem a pleno sol e florescem muito bem no verão, sendo rústicos e de fácil cuidado. Sua haste tem aproximadamente $30 \mathrm{~cm}$ e a largura da flor varia entre 7 e $8 \mathrm{~cm}$ (PEIXOTO, 2008).

Os amarílis ou açucenas, pertencentes à família Amaryllidaceae, são espécies bulbosas endêmicas da grande Bacia Amazônica. Seu bulbo é importante produto florícola no mercado mundial, contudo, as hastes florais têm menor expressão econômica. As variedades cultivadas são normalmente tetraplóides, fruto de cruzamentos iniciados na Europa no século 19, envolvendo diversas espécies botânicas (TOMBOLATO, 2004). O Hippeastrum papilio (figura 1B), também conhecido fora do Brasil como "butterfly amaryllis", é originário do Rio Grande do Sul. As hastes florais medem cerca de $50 \mathrm{~cm}$ e, normalmente, na extremidade abrem-se 2 flores, cada uma medindo cerca de $15 \mathrm{~cm}$ de diâmetro. Suas flores possuem listras em um degradê de cores do centro para fora, com o fundo brancoesverdeado; seu florescimento se inicia no mês de junho, preferencialmente em solo do tipo arenoso. Ao contrário dos outros amarílis, este não perde suas folhas, que permanecem sempre verdes. O Hippeastrum glaucescens (figura 1C) é um dos mais comuns no Brasil, com flores avermelhadas, rosadas, esbranquiçadas ou esverdeadas com uma reticulação avermelhada na parte superior das tépalas; suas flores medem cerca de $15 \mathrm{~cm}$ e se abrem aos pares em hastes de $50 \mathrm{~cm}$ de altura; floresce no inverno e o melhor solo para seu cultivo é do tipo pedregoso (PEIXOTO, 2008).

A coleção do Jardim Botânico do Instituto Agronômico - JBIAC - é provavelmente uma das mais completas do País e ali há também preocupação com a perpetuação das espécies com potencial ornamental, como é o caso das espécies da família Amaryllidaceae, cujos híbridos, conhecidos internacionalmente como "amaryllis" são importantes variedades no comércio mundial. Para isso, a crioconservação pode ser uma ferramenta importante, e por esse motivo estabeleceu-se este experimento.

\section{MATERIAL E MÉTODOS}

Foram usados acessos de três espécies de Amaryllidaceae: Hippeastrum papilio (HP), Hippeastrum glaucescens (HG) e Zephyranthes robusta (ZR). As sementes de HP e ZR foram obtidas a partir de plantas cultivadas na coleção do JBIAC; já as sementes de HG foram colhidas

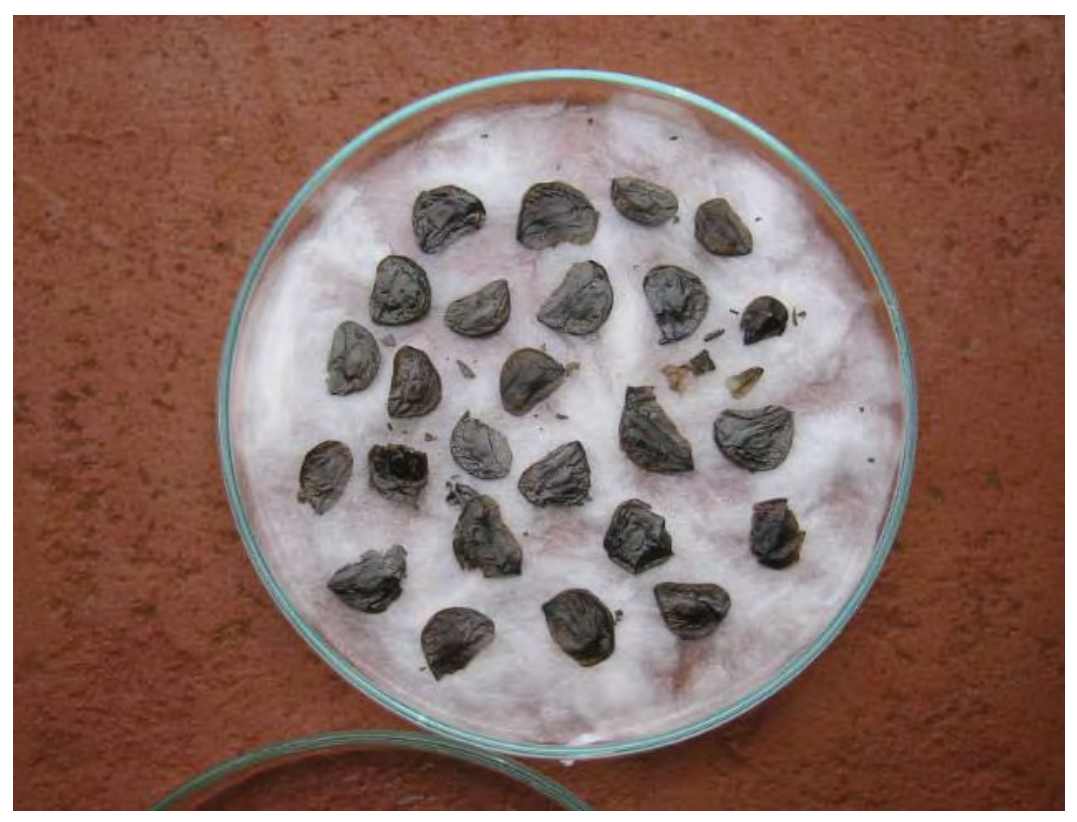

Figura 2. Placa de Petri contendo uma camada de algodão umedecido sobre a qual foi observada a germinação de sementes de Hippeastrum glaucescens. 
em população natural no Pico das Cabras (Campinas, SP). Realizaram-se quatro tratamentos para as espécies de Amaryllidaceae:

1 - crioconservação a $-1960 \mathrm{C}$ sem protetor;

2 - crioconservação a $-1960 \mathrm{C}$ com protetor de alginato (0,25 $\mathrm{ml}$ de alginato de sódio a $1 \%$ durante 20 minutos em solução de cloreto de cálcio $0,1 \mathrm{Mol}-\mathrm{CaCl} 2=2,19$ $\mathrm{g} / 100 \mathrm{ml}$ );

3 - crioconservação a -196oC com protetor PVS2 (30 ml de glicerol, $15 \mathrm{ml}$ de etilenoglicol, 13,69 $\mathrm{g}$ de sacarose, $40 \mathrm{ml}$ de água destilada para $100 \mathrm{ml}$ de solução);

4 - testemunha.

As sementes foram colocadas em recipientes contendo os crioconservadores de forma que todas ficassem recobertas; em seguida, foram embaladas em papel alumínio e submetidas a congelamento rápido com nitrogênio líquido $\left(-196^{\circ} \mathrm{C}\right)$, no qual foram mantidas por no mínimo 24 horas. Após esse período, foram retiradas dos tambores e descongeladas rapidamente. Em cada tratamento, foi analisada uma amostra de 25 sementes em 4 repetições.

A germinação foi realizada em placas de Petri contendo uma camada de algodão umedecido; essas foram mantidas em câmara de cultivo tipo BOD, com temperatura constante de $25^{\circ} \mathrm{C}$ (figura 2). $\mathrm{O}$ efeito da crioconservação na qualidade das sementes foi avaliado pela contagem das sementes germinadas a cada 2 dias. Os dados foram transformados em arc sen $(\sqrt{ } \mathrm{x} / 100)$ e submetidos à análise de variância, com as médias dos fatores qualitativos comparadas pelo teste de Tukey a $5 \%$ de probabilidade.

\section{RESULTADOS E DISCUSSÃO}

A partir dos resultados obtidos (tabela 1), observa-se que a crioconservação de sementes de espécies de Amaryllidaceae é viável, uma vez que as taxas de germinação das sementes crioconservadas foram equivalentes às do método de testemunha. Para HP, a germinação das sementes da testemunha foi de $72 \%$, das crioconservadas sem protetor $70 \%$, e com o protetor alginato de sódio $68 \%$, estatisticamente iguais; para $\mathrm{HG}$, a germinação da testemunha foi de $97 \%$ e da crioconservada sem protetor $88 \%$, estatisticamente diferentes; por fim, para ZR, a germinação da testemunha foi de $100 \%$, da crioconservada sem protetor $99 \%$ e do tratamento com alginato de sódio $95 \%$, estatisticamente iguais. O uso de PVS2 provou a morte da quase totalidade das sementes para as duas espécies de Hippeastrum, com germinação de $1 \%$ das sementes de ZR e $2 \%$ de $\mathrm{HG}$, e afetou sensivelmente a germinação de HP, que caiu para $15 \%$, o único tratamento estatisticamente diferenciado quando comparado aos outros dois e à testemunha.

Pelos dados obtidos no experimento, observa-se que os crioprotetores não foram eficientes para a conservação de sementes dessas três espécies de Amaryllidaceae, com exceção do HP, cujas sementes tratadas com alginato de sódio apresentaram uma porcentagem de germinação estatisticamente igual ao tratamento sem agente protetor. Esses tratamentos com alginato de sódio foram inadequados para todas as espécies testadas, reduzindo significativamente as taxas de germinação. Nos tratamentos com PVS2, também houve queda na taxa de germinação, porém menos significativa do que naqueles.

A crioconservação sem agente protetor, mostrou a germinação mais rápida do que a testemunha para as três espécies testadas. Pelos dados colhidos a cada dois dias, constatou-se uma possível quebra de dormência da semente para ZR, pois houve uma aceleração no processo de germinação de cerca de dois dias. Por tratar-se de uma espécie cujas sementes apresentam alto poder germinativo e em curto espaço de tempo, essa observação merece maior aprofundamento.

Tabela 1. Médias de porcentagens e médias transformadas em arc sen $\sqrt{\mathrm{X}} / 100$ da germinação de sementes das espécies Zephyranthes robusta (ZR), Hippeastrum glaucescens (HG) e Hippeastrum papilio (HP) submetidas a três tratamentos de crioconservação.

\begin{tabular}{|c|c|c|c|c|c|c|}
\hline \multirow{3}{*}{ Tratamento } & \multicolumn{6}{|c|}{ Espécie } \\
\hline & \multicolumn{2}{|c|}{ ZR } & \multicolumn{2}{|c|}{ HG } & \multicolumn{2}{|c|}{ HP } \\
\hline & $\begin{array}{l}\operatorname{arcsen} \\
\sqrt{\mathrm{x}} / 100\end{array}$ & $\%$ & $\begin{array}{l}\operatorname{arcsen} \\
\sqrt{\mathrm{x}} / 100\end{array}$ & $\%$ & $\begin{array}{l}\operatorname{arcsen} \\
\sqrt{\mathrm{x}} / 100\end{array}$ & $\%$ \\
\hline$-196^{\circ} \mathrm{C}$ & 1,5707 & $100 \mathrm{a}$ & 1,0774 & $88 \mathrm{~b}$ & 0,7766 & $70 \mathrm{a}$ \\
\hline $196^{\circ} \mathrm{C}+$ Alginato de sódio & 1,2523 & $95 \mathrm{~b}$ & 0,3718 & $36 \mathrm{c}$ & 0,7538 & $68 \mathrm{a}$ \\
\hline $196^{\circ} \mathrm{C}+\mathrm{PVS} 2$ & 0,01007 & $1 \mathrm{c}$ & 0,02 & $2 \mathrm{~d}$ & 0,1511 & $15 \mathrm{~b}$ \\
\hline testemunha & 1,4288 & $99 \mathrm{ab}$ & 1,3984 & $97 \mathrm{a}$ & 0,8124 & $73 \mathrm{a}$ \\
\hline Dms & \multicolumn{2}{|c|}{0,2965} & \multicolumn{2}{|c|}{0,3054} & \multicolumn{2}{|c|}{0,2073} \\
\hline
\end{tabular}




\section{CONCLUSÃ̃}

A crioconservação é uma técnica que pode ser aplicada com sucesso para a conservação em longo prazo de sementes das espécies de Amaryllidaceae testadas; porém, o uso das substâncias crioprotetoras alginato de sódio e PVS2 não alterou a germinação das sementes, em relação às não tratadas.

\section{REFERÊNCIAS}

ASHWOOD-SMITH, M.J. Genetic damage is not produced by normal cryopreservation involving either glycerol or dimethyl sulphoxide: a cautionary note, however, on possible effects of dimethyl sulphoxide. Cryobiology, v.22, p.427-433, 1985 .

ELLIS, R.H; HONG, T.D; ROBERTS, E.H. An intermediate category of seed storage behavior? Journal of Experimental Botany, v. 41, n. 9, p. 1167-1174, 1990.

ENGELMANN, F. In vitro conservation of tropical plant germoplasm - a review. Euphytica, 57:227-243, 1991.

FONSECA, S. C. L; FREIRE, H. B. Sementes recalcitran- tes: problemas na pós-colheita. Bragantia, Campinas, v. 62, n. 2, 2003.

GONZÁLES-BENITO, M.E. Cryopreservation as a tool for preserving genetic variability: its use with Spanish wild species with possible landscaping value. Acta Horticulturae, Leuven, v.457, p. 133-142, 1998.

PEIXOTO, M. Zephyranthes robusta.

Disponível em: http://mpeixoto.sites.uol.com.br/amaryl/ robusta.html. acesso em: 8 de outubro de 2008 .

ROBERTS, E.H. Predicting the storage life of seeds. Seed Science and Technology, v.1, p.499-514, 1973.

SANTOS, I.R.I. Crioconservação: potencial e perspectivas para a conservação de germoplasma vegetal. Revista Brasileira de Fisiologia Vegetal, v. 12, n (especial), p.70-84, 2000.

TOMBOLATO, A.F.C. Cultivo comercial de plantas ornamentais. Campinas: Instituto Agronômico. 2004. 211p.

TORRES, A. C.; CALDAS, L. S.; BUSO, J. A. Cultura de tecidos e transformação genética de plantas. Brasília: Embrapa-SPI/ Embrapa-CNPH, v. 1 e 2. 1998. 314p. 
